\title{
Implementing Mediators with Asynchronous Cheap Talk
}

\author{
Ittai Abraham \\ ittaia@cs.huji.ac.il \\ VMWARE \\ Ivan Geffner $^{\dagger}$
ieg8@cornell.edu
Cornell University
}

\begin{abstract}
A mediator can help non-cooperative agents obtain an equilibrium that may otherwise not be possible. We study the ability of players to obtain the same equilibrium without a mediator, using only cheap talk, that is, nonbinding pre-play communication. Previous work has considered this problem in a synchronous setting. Here we consider the effect of asynchrony on the problem, and provide upper bounds for implementing mediators. Considering asynchronous environments introduces new subtleties, including exactly what solution concept is most appropriate and determining what move is played if the cheap talk goes on forever. Different results are obtained depending on whether the move after such "infinite play" is under the control of the players or part of the description of the game.
\end{abstract}

\section{CCS CONCEPTS}

- Security and privacy $\rightarrow$ Formal security models; Distributed systems security; $\bullet$ Theory of computation $\rightarrow$ Algorithmic mechanism design; Solution concepts in game theory; • Networks $\rightarrow$ Network security;

\section{KEYWORDS}

distributed computing, game theory, security

\section{ACM Reference Format:}

Ittai Abraham, Danny Dolev, Ivan Geffner, and Joseph Y. Halpern. 2019. Implementing Mediators with Asynchronous Cheap Talk. In 2019 ACM Symposium on Principles of Distributed Computing (PODC '19), July 29August 2, 2019, Toronto, ON, Canada. ACM, New York, NY, USA, 10 pages. https://doi.org/10.1145/3293611.3331623

\footnotetext{
*Supported by The Federmann Cyber Security Center in conjunction with the Israe National Cyber Directorate.

${ }^{\dagger}$ Supported in part by NSF grant IIS-1703846.

¥Supported in part by NSF grants IIS-1703846 and IIS-1718108, ARO grant W911NF17-1-0592, and a grant from Open Philanthropy.
}

Permission to make digital or hard copies of all or part of this work for personal or classroom use is granted without fee provided that copies are not made or distributed for profit or commercial advantage and that copies bear this notice and the full citation on the first page. Copyrights for components of this work owned by others than the author(s) must be honored. Abstracting with credit is permitted. To copy otherwise, or republish, to post on servers or to redistribute to lists, requires prior specific permission and/or a fee. Request permissions from permissions@acm.org.

PODC '19, July 29-August 2, 2019, Toronto, ON, Canada

(c) 2019 Copyright held by the owner/author(s). Publication rights licensed to ACM. ACM ISBN 978-1-4503-6217-7/19/07 ..\$15.00

https://doi.org/10.1145/3293611.3331623

\author{
Danny Dolev ${ }^{*}$ \\ dolev@cs.huji.ac.il \\ The Hebrew University of Jerusalem \\ Jerusalem, Israel \\ Joseph Y. Halpern ${ }^{\ddagger}$
halpern@cs.cornell.edu
Cornell University
}

\section{INTRODUCTION}

Having a trusted mediator often makes solving a problem much easier. For example, a problem such as Byzantine agreement becomes trivial with a mediator: agents can just send their initial input to the mediator, and the mediator sends the majority value back to all the agents, which they then output. Not surprisingly, the question of whether a problem in a multiagent system that can be solved with a trusted mediator can be solved by just the agents in the system, without the mediator, has attracted a great deal of attention in both computer science (particularly in the cryptography community) and game theory. In cryptography, the focus has been on secure multiparty computation $[12,15]$. Here it is assumed that each agent $i$ has some private information $x_{i}$. Fix functions $f_{1}, \ldots, f_{n}$. The goal is to have agent $i$ learn $f_{i}\left(x_{1}, \ldots, x_{n}\right)$ without learning anything about $x_{j}$ for $j \neq i$ beyond what is revealed by the value of $f_{i}\left(x_{1}, \ldots, x_{n}\right)$. With a trusted mediator, this is trivial: each agent $i$ just gives the mediator its private value $x_{i}$; the mediator then sends each agent $i$ the value $f_{i}\left(x_{1}, \ldots, x_{n}\right)$. Work on multiparty computation provides conditions under which this can be done in a synchronous system $[7,12,14,15]$ and in an asynchronous system $[6,8]$. In game theory, the focus has been on whether an equilibrium in a game with a mediator can be implemented using what is called cheap talk-that is, just by players communicating among themselves.

In the computer science literature, the interest has been in performing multiparty computation in the presence of possibly malicious adversaries, who do everything they can to subvert the computation. In contrast, in the game theory literature, the assumption is that players have preferences and seek to maximize their utility; thus, they will subvert the computation iff it is in their best interests to do so. In $[2,3]$ (denoted $\mathrm{ADGH}$ and $\mathrm{ADH}$, respectively, in the rest of the paper), it was argued that it is important to consider deviations by both rational players, who have preferences and try to maximize them, and players that we can view as malicious, although it is perhaps better to think of them as rational players whose utilities are not known by the mechanism designer (or other players). $\mathrm{ADGH}$ and $\mathrm{ADH}$ considered equilibria that are $(k, t)$-robust; roughly speaking, this means that the equilibrium tolerates deviations by up to $k$ rational players, whose utilities are presumed known, and up to $t$ players with unknown utilities. Tight bounds were proved on the ability to implement a $(k, t)$-robust equilibrium in the game with a mediator using cheap talk in synchronous systems. These bounds depend on, among other things, 
(a) the relationship between $k, t$ and $n$, the total number of players in the system; (b) whether players know the exact utilities of the rational players; and (c) whether the game has a punishment strategy, where an $m$-punishment strategy is a strategy profile that, if used by all but at most $m$ players, guarantees that every player gets a worse outcome than they do with the equilibrium strategy. The following is a high-level overview of results proved in the synchronous setting that will be of most relevance here. For these results, we assume that the communication with the mediator is bounded, it lasts for at most $N$ rounds, and that the mediator can be represented by an arithmetic circuit of depth $c$.

R1. If $n>3 k+3 t$, then a mediator can be implemented using cheap talk; no punishment strategy is required, no knowledge of other agents' utilities is required, and the cheap-talk protocol has bounded running time $O(n N c)$, independent of the utilities.

R2. If $n>2 k+3 t$, then a mediator can be implemented using cheap talk if there is a $(k+t)$-punishment strategy and the utilities of the rational players are known; the cheap-talk protocol has expected running time $O(n N c)$. (In R2, unlike $\mathrm{R} 1$, the cheap-talk protocol may be unbounded, although it has finite expected running time.)

In $\mathrm{ADH}$, lower bounds are presented that match the upper bounds above. Thus, for example, it is shown that $n>3 k+3 t$ is necessary in $\mathrm{R} 1$; if $n \leq 3 k+3 t$, then we cannot implement a mediator in general if we do not have a punishment strategy or if the utilities are unknown. The proofs of the upper bounds make heavy use of the fact that the setting is synchronous. Here we consider the impact of asynchrony on these results. Once we introduce asynchrony, we must revisit the question of what it even means to implement an equilibrium using cheap talk. Notions like (Bayesian) Nash equilibrium implicitly assume that all uncertainty is described probabilistically. Having a probability is necessary to talk about an agent's expected utility, given that a certain strategy profile is played. If we were willing to put a distribution on how long messages take to arrive and on when agents are scheduled to move, then we could apply notions like Nash equilibrium without difficulty. However, it is notoriously difficult to quantify this uncertainty. The typical approach used to analyze algorithms in the presence of uncertainty that is not quantified probabilistically is to assume that all the non-probabilistic uncertainty is resolved by the environment according to some strategy. Thus, the environment uses some strategy to decide when each agent will be allowed to play and how long each message takes to be delivered. The algorithm is then proved correct no matter what strategy the environment is following in some class of strategies. For example, we might restrict the environment's strategy to being fair, so that every agent eventually gets a chance to move. (See [13] for a discussion of this approach and further references.)

We follow this approach in the context of games. Note that once we fix the environment's strategy, we have an ordinary game, where uncertainty is quantified by probability. In this setting, we consider ex post equilibrium. A strategy is an ex post equilibrium if it is an equilibrium no matter what strategy the environment uses. Ex post equilibrium is a strong notion, but, as we show by example, it can often be attained with the help of a mediator. It is arguably the closest analogue to Nash equilibrium in an asynchronous setting.
Another issue that plays a major role in an asynchronous setting is what happens if the strategies of players result in some players being livelocked, talking indefinitely without making a move in the underlying game, or in some players being deadlocked, waiting indefinitely without moving in the underlying game. We consider two approaches for dealing with this problem. One is called the default-move approach. In this approach, as part of the description of the game, there is a default move for each player which is imposed if that player fails to explicitly make a move in the cheap-talk phase. Aumann and Hart [5] considered a different approach, which we henceforth call the $A H$ approach, where a player's strategy in the underlying game is a function of the (possibly infinite) history of the player in the cheap-talk phase. We can think of this almost as if the player writes a will, describing what he would like to have done (as a function of the history) if the game ends before he has had a chance to move.

We believe that both the AH approach and the default-move approach are reasonable in different contexts. The AH approach makes sense if the agent can leave instructions that will be carried out by an "executor" if the cheap-talk game deadlocks. But if we consider a game-theoretic variant of Byzantine agreement, it seems more reasonable to say that if a malicious agent can prevent an agent from making a move in finite time, the agent should not get a chance to make a move after the cheap-talk phase has ended.

Our results show that, in the worst case, the cost of asynchrony is an extra $k+t$ in the bounds on $n$, but we can sometimes save $k$ or even $k+t$ if there is a punishment strategy or if we are willing to tolerate an $\epsilon$ "error". For example, with both the AH approach and the default-move approach, if the utilities are not known, we can implement a mediator using asynchronous cheap talk if $n>4 k+4 t$. Thus, compared to $\mathrm{R} 1$, we need an extra $k+t$. However, if we are willing to accept a small probability of error, so that rather than implementing the mediator we get only an $\epsilon$-implementation, and are also willing to accept $\epsilon$-(k,t)-robustness (which, roughly speaking, means that players get within $\epsilon$ of the best they could get), then we can do this if $n>3 k+3 t$, again, using both the $\mathrm{AH}$ approach and the default-move approach.

Just as in the synchronous case, we can do better if we assume that there is a punishment strategy and utilities are known (as in R2). Specifically, with the AH approach, we can implement a mediator if $n>3 k+4 t$ (compared to $n>2 k+3 t$ in the synchronous case), and can $\epsilon$-implement a mediator if $n>2 k+3 t$. We use the punishment to deal with deadlock. If a good player is waiting for a message that never arrives, then the waiting player instructs his executor to carry out a punishment in his will. Having a punishment does not seem to help in the default-move approach unless the default move is a punishment; if it is, then we can get the same results as with the $\mathrm{AH}$ approach.

If there is a punishment strategy, these results significantly improve those of Even, Goldreich, and Lempel [10]. They provide a protocol with similar properties, but the expected number of messages sent is $O(1 / \epsilon)$; with a punishment strategy, we show that a bounded number of messages can be sent, with the bound being independent of $\epsilon$. 


\section{DEFINITIONS}

Asynchronous games, mediator games, and cheap talk: We are interested in implementing mediators. Formally, this means we need to consider three games: an underlying game $\Gamma$, an extension $\Gamma_{d}$ of $\Gamma$ with a mediator, and an extension $\Gamma_{C T}$ of $\Gamma$ with (asynchronous) cheap talk. We assume that $\Gamma$ is a normal-form Bayesian game: each player has a type $t$ taken from some type space $\mathcal{T}_{i}$, such that there is a commonly known distribution on $\mathcal{T} \subseteq \mathcal{T}_{1} \times \cdots \times \mathcal{T}_{n}$, the set of types; each player $i$ chooses an action $a \in A_{i}$, the set of actions of agent $i$; player $i$ 's utility $u_{i}$ is determined by the type profile of the players and the actions they take. A strategy for player $i$ in the Bayesian game is just a function $T_{i}$ to $A_{i}$, which tells player $i$ what to do, given his type. If $A=A_{1} \times \cdots \times A_{n}$, then a strategy profile $\vec{\sigma}=\left(\sigma_{1}, \ldots, \sigma_{n}\right)$ can be viewed as a function $\vec{\sigma}: \mathcal{T} \rightarrow \Delta(A)$ (where, as usual, $\Delta(X)$ denotes the set of probability distributions on $X)$.

The basic notions of a game with a mediator, a cheap-talk game, and implementation are standard in the game-theory literature. However, since we consider them in an asynchronous setting, we must modify the definitions somewhat.

We first define asynchronous games. In an asynchronous game, we assume that players alternate making moves with the environmentfirst the environment moves, then a player moves, then the environment moves, and so on. The environment's move consists of choosing a player $i$ to move next and a set of messages in transit to $i$ that will be delivered just before $i$ moves (so that $i$ 's move can depend on the messages $i$ receives). The environment is subject to two constraints: all messages sent must eventually be delivered and, for all times $m$ and players $i$, if $i$ is still playing the game at time $m$, then there must be some time $m^{\prime} \geq m$ that $i$ is chosen to move. We can describe an asynchronous game by a game tree. Associated with each non-leaf node or history is either a player-the player whose move it is at that node-or the environment (note that both the players and the environment can use probabilistic strategies). The nodes where a player $i$ moves are further partitioned into information sets; intuitively, these are nodes that player $i$ cannot tell apart. We assume that the environment has complete information, so that the environment's information sets just consist of the singletons. A strategy for player $i$ is a (possibly randomized) function from $i$ 's information sets to actions; we can similarly define a strategy for the environment. We can essentially view the environment strategy as defining a scheduler (and thus we sometimes refer to an environment strategy as a scheduler).

For our results, we start with an $n$-player Bayesian game $\Gamma$ in normal form (called the underlying game), with $\{1, \ldots, n\}$ being the set of players, and then consider two games that extend $\Gamma$. A game $\Gamma^{\prime}$ extends $\Gamma$ if the players have initial types from the same type space as $\Gamma$, with the same distribution over types; moreover, in each path of the game tree for $\Gamma^{\prime}$, the players send and receive messages, and perform at most one action from $\Gamma$. In a history where each player makes a move from $\Gamma$, each player gets the same utility as in $\Gamma$ (where the utility is a function of the moves made and the types). That leaves open the question of what happens in a complete history of $\Gamma^{\prime}$ where some players do not make a move in $\Gamma$. As we suggested in the introduction, we consider two approaches to dealing with this. In the first approach, we assume that the description of $\Gamma^{\prime}$ includes a function $M_{i}$ for each player $i$ that maps player $i$ 's type to a move in $\Gamma$. In an infinite history $h$ where $i$ has type $t$ and does not make a move in $\Gamma, i$ is viewed as having made move $M_{i}(t)$. We can then define each player's utility in $h$ as above. This is the default-move approach. In the AH approach, we extend the notion of strategy so that $i$ 's strategy in $\Gamma^{\prime}$ also describes what move $i$ makes in the underlying game $\Gamma$ in any infinite history $h$ where $i$ has not made a move in $\Gamma$. In the AH approach, $i$ 's move in $h$ is under $i$ 's control; in the default-move approach, it is not.

Given an underlying Bayesian game $\Gamma$ (which we assume is synchronous-the players move simultaneously), we will be interested in two types of extensions. A mediator game extending $\Gamma$ is an asynchronous game where players can send messages to and receive messages from a mediator (who can be viewed as a trusted third party) as well as making a move in $\Gamma$; "good" or "honest" players do not send messages to each other, but "bad" players (i.e., one of the $k$ rational deviating players or one of the $t$ "malicious" players with unknown utilities) may send messages to each other as well as to the mediator. We assume that the space of possible messages that can be sent in a mediator game is fixed and finite.

In an asynchronous cheap-talk game extending $\Gamma$, there is no mediator. Players send messages to each other via asynchronous channels, as well as making a move in $\Gamma$. We assume that each pair of agents communicates over an asynchronous authenticated private channel, so the adversary cannot eavesdrop on conversations between the players, and players can identify the sender of each message. Finally, we assume that in both the mediator game and the cheap-talk game, when a player is first scheduled, it gets a signal that the game has started (either an external signal from the environment, or a game-related message from another player or the mediator).

Implementation: In the synchronous setting, a strategy profile $\vec{\sigma}^{\prime}$ in a cheap-talk game $\Gamma_{C T}$ extending an underlying game $\Gamma$ implements a strategy $\vec{\sigma}$ in a mediator game $\Gamma_{d}$ extending $\Gamma$ if $\vec{\sigma}$ and $\vec{\sigma}^{\prime}$ correspond to the same strategy in $\Gamma$; that is, they induce the same function from $\mathcal{T}$ to $\Delta(A)$. The notion of implementation is more complicated in an asynchronous setting, because the probability on action profiles also depends on the environment strategy. Because $\Gamma_{C T}$ and $\Gamma_{d}$ are quite different games, the environment's strategies in $\Gamma_{C T}$ are quite different from those in $\Gamma_{d}$. So we now say that $\vec{\sigma}^{\prime}$ implements $\vec{\sigma}$ if the set of distributions on actions profiles in $\Gamma$ induced by $\vec{\sigma}$ and all possible choices of environment strategy is the same as that induced by $\vec{\sigma}^{\prime}$ and all possible choices of environment strategy. More precisely, let $\mathcal{S}_{\Gamma^{\prime}, e}$ and $\mathcal{S}_{\Gamma^{\prime \prime}, e}$ denote the the set of environment strategies in $\Gamma^{\prime}$ and $\Gamma^{\prime \prime}$, respectively. A strategy $\sigma_{e} \in \mathcal{S}_{\Gamma^{\prime}, e}$ and a strategy profile $\vec{\sigma}$ for the players in $\Gamma^{\prime}$ together induce a function $\left(\vec{\sigma}, \sigma_{e}\right)$ from $\mathcal{T}$ to $\Delta(A)$. A strategy profile $\vec{\sigma}^{\prime}$ in $\Gamma^{\prime}$ implements a strategy profile $\vec{\sigma}^{\prime \prime}$ in $\Gamma^{\prime \prime}$ if $\left\{\left(\vec{\sigma}^{\prime}, \sigma_{e}^{\prime}\right): \sigma_{e}^{\prime} \in \mathcal{S}_{\Gamma^{\prime}, e}\right\}=\left\{\left(\vec{\sigma}^{\prime \prime}, \sigma_{e}^{\prime \prime}\right): \sigma_{e}^{\prime \prime} \in \mathcal{S}_{\Gamma^{\prime \prime}, e}\right\}$. Since the outcome that arises if the players use a particular strategy may depend on what the environment does, this says that the set of outcomes that can result if the players use $\vec{\sigma}^{\prime}$ is the same as the set of outcomes that can result if the players use $\vec{\sigma}^{\prime \prime}$.

For some of our results, we cannot get an exact implementation; there may be some error. Given two discrete distributions $\pi$ and $\pi^{\prime}$ on some space $S$, the distance between $\pi$ and $\pi^{\prime}$, denoted $\operatorname{dist}\left(\pi, \pi^{\prime}\right)$, is at most $\epsilon$ if $\sum_{s \in S}\left|\pi(s)-\pi^{\prime}(s)\right| \leq \epsilon$. As we observed earlier, in 
the mediator game and the cheap-talk game, a strategy profile $\vec{\sigma}$ for the players and a strategy $\sigma_{e}$ for the environment together induce a mapping from type profiles to $\Delta(A)$. We lift the notion of distance to such function by defining $\left.\operatorname{dist}\left(\left(\vec{\sigma}, \sigma_{e}\right)\right),\left(\vec{\sigma}^{\prime}, \sigma_{e}^{\prime}\right)\right)=$ $\max _{\vec{x} \in \mathcal{T}} \operatorname{dist}\left(\left(\vec{\sigma}, \sigma_{e}\right)(\vec{x}),\left(\vec{\sigma}^{\prime}, \sigma_{e}^{\prime}\right)(\vec{x})\right)$. Say that $\vec{\sigma}^{\prime} \epsilon$-implements $\vec{\sigma}^{\prime \prime}$ if

- for all $\sigma_{e}^{\prime} \in \mathcal{S}_{\Gamma^{\prime}, e}$ there exists $\sigma_{e}^{\prime \prime} \in \mathcal{S}_{\Gamma^{\prime \prime}, e}$ such that $\operatorname{dist}\left(\left(\vec{\sigma}^{\prime}, \sigma_{e}^{\prime}\right),\left(\overrightarrow{\sigma^{\prime \prime}}, \sigma_{e}^{\prime \prime}\right)\right) \leq \epsilon$; and

- for all $\sigma_{e}^{\prime \prime} \in \mathcal{S}_{\Gamma^{\prime \prime}, e}$ there exists $\sigma_{e}^{\prime} \in \mathcal{S}_{\Gamma^{\prime}, e}$ such that $\operatorname{dist}\left(\left(\vec{\sigma}^{\prime \prime}, \sigma_{e}^{\prime \prime}\right),\left(\vec{\sigma}^{\prime}, \sigma_{e}^{\prime}\right)\right) \leq \epsilon$.

Note that $\vec{\sigma}^{\prime}$ implements $\vec{\sigma}^{\prime \prime}$ iff $\vec{\sigma}^{\prime} 0$-implements $\vec{\sigma}^{\prime \prime}$.

The notion of implementation is quite strong. For example, if $\vec{\sigma}^{\prime}$ involves fewer rounds of communication than $\vec{\sigma}^{\prime \prime}$, there may be far fewer distinct schedulers in the game involving $\vec{\sigma}^{\prime}$ than in the game involving $\vec{\sigma}^{\prime \prime}$. Thus, we may not be able to recover the effect of all possible schedulers. (Indeed, for some of our results the implementation needs to be quite long precisely in order to capture all possible schedulers.) This suggests the following notion: a strategy profile $\vec{\sigma}^{\prime}$ in $\Gamma^{\prime}$ weakly implements a strategy profile $\vec{\sigma}^{\prime \prime}$ in $\Gamma^{\prime \prime}$ if $\left\{\left(\vec{\sigma}^{\prime}, \sigma_{e}^{\prime}\right): \sigma_{e}^{\prime} \in \mathcal{S}_{\Gamma^{\prime}, e}\right\} \subseteq\left\{\left(\vec{\sigma}^{\prime \prime}, \sigma_{e}^{\prime \prime}\right): \sigma_{e}^{\prime \prime} \in \mathcal{S}_{\Gamma^{\prime \prime}, e}\right\}$. Thus, if $\vec{\sigma}^{\prime}$ weakly implements $\vec{\sigma}^{\prime \prime}$, then every outcome of $\vec{\sigma}^{\prime}$ is one that could also have arisen with $\vec{\sigma}^{\prime \prime}$, but the converse may not be true. Specifically, there may be some behaviors of the environment with $\vec{\sigma}^{\prime \prime}$ that cannot be simulated by $\vec{\sigma}^{\prime}$. As we shall see, this may actually be a feature: we can sometimes simulate the effect of only "good" schedulers. In any case, note that in the synchronous setting, implementation and weak implementation coincide. We can also define a notion of weak $\epsilon$-implementation in the obvious way; we leave the details to the reader.

Termination: We will be interested in asynchronous games where, almost surely, the honest players stop sending messages and make a move in the underlying game. In the mediator games that we consider, this happens after only a bounded number of messages have been sent. But even with this bound, there may not be a point in a history when players know that they can stop sending messages; although a player $i$ may have moved in the underlying game, $i$ may still need to keep checking for incoming message, and may need to respond to them, to ensure that other players can make the appropriate move.

For some of our results, we must assume that, in the mediator game, there comes a point when all honest players know that they have terminated the protocol; they will not get further messages from the mediator and can stop sending messages to the mediator, and should make a move in the underlying game if they have not done so yet. For simplicity, for these results, we restrict the honest players and the mediator to using strategy profiles that have the following canonical form: Using a canonical strategy, player $i$ may send a message to the mediator whenever it is scheduled, as long as $i$ has not received a message from the mediator containing "STOP". If player $i$ gets a message from the mediator that includes "STOP", then $i$ makes a move in the underlying game and halts. We assume that, as long as the honest players and mediator follow their part of the canonical strategy profile, there is a constant $r$ such that, no matter what strategy the rational and malicious players and the environment use, the mediator sends each player $i$ at most $r$ messages in each history, and the final message includes "STOP". We conjecture that the assumption that players and mediator are using a strategy in canonical form in the mediator game is without loss of generality; that is, a $(k, t)$-robust strategy profile in a mediator game $\Gamma_{d}$ can be implemented by a $(k, t)$-robust strategy profile in $\Gamma_{d}$ that is in canonical form.

\section{SOLUTION CONCEPTS}

In this section, we review the solution concepts introduced in ADGH and extend them to asynchronous settings.

Note that in an asynchronous game $\Gamma$, the utility of a player $i$ can depend not only on the strategies of the agents, but on what the environment does. Since we consider an underlying game, a mediator game, and a cheap-talk game, it is useful to include explicitly in the utility function which game is being considered. Thus, we write $u_{i}\left(\Gamma, \vec{\sigma}, \sigma_{d}, \sigma_{e}, \vec{x}\right)$ to denote the expected utility of player $i$ in game $\Gamma$ when players play strategy profile $\vec{\sigma}$, the mediator plays $\sigma_{d}$, the environment plays $\sigma_{e}$, and the type profile is $\vec{x}$. We typically say "input profile" rather than "type profile", since in our setting, the type of player $i$ is just $i$ 's initial input. Note that if $\Gamma$ is the underlying game, the $\sigma_{e}$ component is unnecessary, since the underlying game is assumed to be synchronous. We occasionally omit the mediator strategy $\sigma_{d}$ when it is clear from context.

Given a type space $\mathcal{T}$, a set $K$ of players, and $\vec{x} \in \mathcal{T}$, let $\mathcal{T}\left(\vec{x}_{K}\right)=$ $\left\{\vec{x}^{\prime}: \vec{x}_{K}^{\prime}=\vec{x}_{K}\right\}$. If $\Gamma$ is a Bayesian game over type space $\mathcal{T}, \vec{\sigma}$ is a strategy profile in $\Gamma$, and $\operatorname{Pr}$ is the probability on the type space $\mathcal{T}$, let

$$
u_{i}\left(\Gamma, \vec{\sigma}, \sigma_{e}, \vec{x}_{K}\right)=\sum_{\vec{x}^{\prime} \in \mathcal{T}\left(\vec{x}_{K}\right)} \operatorname{Pr}\left(\vec{x}^{\prime} \mid \mathcal{T}\left(\vec{x}_{K}\right)\right) u_{i}\left(\Gamma, \vec{\sigma}, \sigma_{e}, \vec{x}^{\prime}\right) .
$$

Thus, $u_{i}\left(\Gamma, \vec{\sigma}, \sigma_{e}, \vec{x}_{K}\right)$ is $i$ 's expected payoff if everyone uses strategy $\vec{\sigma}$ and type profiles are in $\mathcal{T}\left(\vec{x}_{K}\right)$.

$k$-resilient equilibrium: In a standard game, a strategy profile is a Nash equilibrium if no player can gain any advantage by using a different strategy, given that all the other players do not change their strategies. The notion of $k$-resilient equilibrium extends Nash equilibrium to allow for coalitions.

Definition 3.1. $\vec{\sigma}$ is a $k$-resilient equilibrium (resp., strongly $k$-resilient equilibrium) in an asynchronous game $\Gamma$ if, for all subsets $K$ of players with $1 \leq|K| \leq k$, all strategy profiles $\vec{\tau}_{K}$ for the players in $K$, all type profiles $\vec{x} \in \mathcal{T}$, and all strategies $\sigma_{e}$ of the environment, $u_{i}\left(\Gamma,\left(\vec{\sigma}_{-K}, \vec{\tau}_{K}\right), \sigma_{e}, \vec{x}_{K}\right) \leq u_{i}\left(\Gamma, \vec{\sigma}, \sigma_{e}, \vec{x}_{K}\right)$ for some (resp., all) $i \in$ $K^{1}$

Thus, $\vec{\sigma}$ is $k$-resilient if, no matter what the environment does, no subset $K$ of at most $k$ players can all do better by deviating, even if they share their type information (so that if the true type is $\vec{x}$, the players in $K$ know $\vec{x}_{K}$ ). It is strongly $k$-resilient if not even one of the players in $K$ can do better if all the players in $K$ deviate.

For some of our results we will be interested in equilibria that are "almost" $k$-resilient, in the sense that no player in a coalition can do more than $\epsilon$ better if the coalition deviates from the protocol, for some small $\epsilon$.

Definition 3.2. For $\epsilon>0, \vec{\sigma}$ is an $\epsilon$ - $k$-resilient equilibrium (resp., strongly $\epsilon$ - $k$-resilient equilibrium) if, for all subsets $K$ of players, all

\footnotetext{
${ }^{1}$ As usual, in the strategy profile $\left(\vec{\sigma}_{-K}, \vec{\tau}_{K}\right)$, each player $i \in K$ plays $\tau_{i}$ and each player $i \notin K$ plays $\sigma_{i}$.
} 
strategy profiles $\vec{\tau}_{K}$ for the players in $K$, all type profiles $\vec{x} \in \mathcal{T}$, and all strategies $\sigma_{e}$ of the environment, we have $u_{i}\left(\Gamma,\left(\vec{\sigma}_{-K}, \vec{\tau}_{K}\right), \sigma_{e}, \vec{x}_{K}\right)<$ $u_{i}\left(\Gamma, \vec{\sigma}, \sigma_{e}, \vec{x}_{K}\right)+\epsilon$ for some (resp., for all) $i \in K$.

Note that we have " $<u_{i}\left(\Gamma, \vec{\sigma}, \sigma_{e}, \vec{x}_{K}\right)+\epsilon$ " here, not " $\leq$ "; this means that a $0-k$-resilient equilibrium is not a $k$-resilient equilibrium. However, an equilibrium is $k$-resilient iff it is $\epsilon$ - $k$-resilient for all $\epsilon>0$. We have used this slightly nonstandard definition to make the statements of our theorems cleaner.

Robustness: A standard assumption in game theory is that utilities are (commonly) known; when we are given a game we are also given each player's utility. When players make decision, they can take other players' utilities into account. However, in large systems, it seems almost invariably the case that there will be some fraction of users who do not respond to incentives the way we expect. For example, in a peer-to-peer network like Kazaa or Gnutella, it would seem that no rational agent should share files. Whether or not you can get a file depends only on whether other people share files; on the other hand, it seems that there are disincentives for sharing (the possibility of lawsuits, use of bandwidth, etc.). Nevertheless, people do share files. However, studies of the Gnutella network have shown almost 70 percent of users share no files and nearly 50 percent of responses are from the top 1 percent of sharing hosts [4]. It seems important to design protocols that tolerate such unanticipated behaviors, so that the payoffs of the users who follow the recommended strategy are not affected by players who deviate, provided that not too many deviate.

Definition 3.3. A strategy profile $\vec{\sigma}$ is $t$-immune in a game $\Gamma$ if, for all subsets $T$ of players with $|T| \leq t$, all strategy profiles $\vec{\tau}$, all $i \notin T$, all type profiles $\vec{x} \in \mathcal{T}$, and all strategies $\sigma_{e}$ of the environment, we have $u_{i}\left(\Gamma,\left(\vec{\sigma}_{-T}, \vec{\tau}_{T}\right), \sigma_{e}, \vec{x}_{T}\right) \geq u_{i}\left(\Gamma, \vec{\sigma}, \sigma_{e}, \vec{x}_{T}\right)$.

Intuitively, $\vec{\sigma}$ is $t$-immune if there is nothing that players in a set $T$ of size at most $t$ can do to give the players not in $T$ a worse payoff, even if the players in $T$ share their type information.

The notion of $t$-immunity and $k$-resilience address different concerns. For $t$-immunity, we consider the payoffs of the players not in $K$; for $k$-resilience, we consider the payoffs of players in $K$. It is natural to combine both notions. Given a strategy profile $\vec{\tau}$, let $\Gamma_{\vec{\tau}}^{T}$ be the game which is identical to $\Gamma$ except that the players in $T$ are fixed to playing strategy $\vec{\tau}_{T}$.

Definition 3.4. $\vec{\sigma}$ is a (strongly) $(k, t)$-robust equilibrium in a game $\Gamma$ if $\vec{\sigma}$ is t-immune and, for all subsets $T$ of players with $|T| \leq t$ and all strategy profiles $\vec{\tau},\left(\vec{\sigma}_{-T}, \vec{\tau}_{T}\right)$ is a (strongly) $k$-resilient equilibrium of $\Gamma_{\vec{\tau}}^{T}$.

We can define "approximate" notions of $t$-immunity and $(k, t)$ robustness analogous to Definition 3.2:

Definition 3.5. For $\epsilon>0$, a strategy profile $\vec{\sigma}$ is $\epsilon$ - $t$-immune in $\Gamma$ if, for all subsets $T$ of players with $|T| \leq t$, all strategy profiles $\vec{\tau}$, all $i \notin T$, all type profiles $\vec{x} \in \mathcal{T}$, and all strategies $\sigma_{e}$ of the environment, we have $u_{i}\left(\Gamma,\left(\vec{\sigma}_{-T}, \vec{\tau}_{T}\right), \sigma_{e}, \vec{x}_{T}\right)>u_{i}\left(\Gamma, \vec{\sigma}, \sigma_{e}, \vec{x}_{T}\right)-\epsilon$.

Definition 3.6. For $\epsilon \geq 0, \vec{\sigma}$ is a (strongly) $\epsilon$ - $(k, t)$-robust equilibrium in $\Gamma$ if $\vec{\sigma}$ is $\epsilon$-t-immune and, for all subsets $T$ of players with $|T| \leq t$ and strategy profiles $\vec{\tau}_{T},\left(\vec{\sigma}_{-T}, \vec{\tau}_{T}\right)$ is a (strongly) $\epsilon-k$-resilient equilibrium of $\Gamma_{\vec{\tau}}^{T}$.

\section{MAIN THEOREMS: FORMAL STATEMENTS}

In this section, we state our results formally. We begin with a result that is an analogue of R1 in the asynchronous setting. We say that a game $\Gamma^{\prime}$ is a utility variant of a game $\Gamma$ if $\Gamma^{\prime}$ and $\Gamma$ have the same game tree, but the utilities of the players may be different in $\Gamma$ and $\Gamma^{\prime}$. We use the notation $\Gamma(\vec{u})$ if we want to emphasize that $\vec{u}$ is the utility function in game $\Gamma$. We then take $\Gamma\left(\vec{u}^{\prime}\right)$ to be the utility variant of $\Gamma$ with utility function $\vec{u}^{\prime}$.

Two more technical comments before stating the theorems: in the mediator game we can also view the mediator as a player (albeit one without a utility function) that is following a strategy. Thus, when we talk about a strategy profile that is a $(k, t)$-robust equilibrium in the mediator game, we must give the mediator's strategy as well as the players' strategies. We sometimes write $\vec{\sigma}+\sigma_{d}$ if we want to distinguish the players' strategy profile $\vec{\sigma}$ from the mediator's strategy $\sigma_{d}$. We occasionally abuse notation and drop the $\sigma_{d}$ if it is clear from context, and just talk about $\vec{\sigma}$ being a $(k, t)$-robust equilibrium.

In general, we do not have a bound on the number of messages sent in our implementation. However, we can get a polynomial bound if we restrict the mediator to using what we call a responsive strategy. Roughly speaking, a responsive strategy is one where (1) the mediator sends messages only initially or in response to receiving a message since the last time it moved; (2) if it has received a message since the last time it was scheduled, it acts just as it would have acted if the message had been received immediately after the last time it acted (rather than possibly being received after a number of times that the mediator was scheduled without receiving a new message); and (3) there is a bound $C$ such that the mediator uses at most $C$ random bits in each run. see [11] for the formal definition.

THEOREM 4.1. If $\Gamma$ is a normal-form Bayesian game with $n$ players, $\vec{\sigma}+\sigma_{d}$ is a strategy profile for the players and the mediator in an asynchronous mediator game $\Gamma_{d}$ that extends $\Gamma$, and $n>4 k+4 t$, then with both the default-move approach and the AH approach, there exists a strategy profile $\vec{\sigma}_{C T}$ that implements $\vec{\sigma}+\sigma_{d}$ in the asynchronous cheap-talk game $\Gamma_{C T}$ such that for all utility variants $\Gamma_{d}\left(\vec{u}^{\prime}\right)$ of $\Gamma_{d}$, if $\vec{\sigma}+\sigma_{d}$ is a (strongly) $(k, t)$-robust equilibrium in $\Gamma_{d}\left(\vec{u}^{\prime}\right)$, then $\vec{\sigma}_{C T}$ is a (strongly) $(k, t)$-robust equilibrium in $\Gamma_{C T}\left(\vec{u}^{\prime}\right)$. If $\sigma_{d}$ is responsive, the number of messages sent in a history of $\vec{\sigma}_{C T}$ is polynomial in $n$ and $N$, linear in $c$, and independent of $\vec{u}^{\prime}$.

The proof of Theorem 4.1 uses ideas from the multiparty computation protocol of Ben-Or, Canetti, and Goldreich [6] (BCG from now on). Our construction actually needs stronger properties than these provided by BCG; we show that we can get protocols with these stronger properties in a companion paper [11]; see Section 5 for further discussion.

We can obtain better bounds if we are willing to accept $\epsilon$-equilibrium, using ideas due to Ben-Or, Kelmer, and Rabin [8].

THEOREM 4.2. If $\Gamma$ is a normal-form Bayesian game with $n$ players, $\vec{\sigma}+\sigma_{d}$ is a strategy profile for the players and mediator in an asynchronous mediator game $\Gamma_{d}$ that extends $\Gamma, M>0$, and $n>3 k+3 t$, then with both the default-move approach and the AH approach, for all $\epsilon>0$, there exists a strategy profile $\vec{\sigma}_{C T}$ in the asynchronous cheap-talk game $\Gamma_{C T}$ that $\epsilon$-implements $\vec{\sigma}$ such that for all utility variants $\Gamma_{d}\left(\vec{u}^{\prime}\right)$ of $\Gamma_{d}$ bounded by $M / 2$ (i.e., where the range of $u_{i}^{\prime}$ 
is contained in $[-M / 2, M / 2])$, if $\vec{\sigma}+\sigma_{d}$ is a (strongly) $(k, t)$-robust equilibrium in $\Gamma_{d}\left(\vec{u}^{\prime}\right)$, then $\vec{\sigma}_{C T}$ is a (strongly) $\epsilon-(k, t)$-robust equilibrium in $\Gamma_{C T}\left(\vec{u}^{\prime}\right)$. If $\sigma_{d}$ is responsive, the number of messages sent in a history of $\vec{\sigma}_{C T}$ is polynomial in $n$ and $N$, linear in $c$, and independent of $\vec{u}^{\prime}$.

If we have a punishment strategy and utilities are known, we can do better with the AH approach. To make this precise, we need the definition of an $m$-punishment strategy [2] (which generalizes the notion of punishment strategy defined by Ben Porath [9]). Before defining this carefully, note that in an asynchronous setting (i.e., in the mediator game and the cheap-talk game, but not in the underlying game), the utility of players depends on the environment's strategy as well as the players' strategy profile and the players' type profile.

Definition 4.3. If $\Gamma^{\prime}$ is an extension of an underlying game $\Gamma$, a strategy profile $\vec{\rho}$ in $\Gamma$ is a $k$-punishment strategy with respect to a strategy profile $\vec{\sigma}^{\prime}$ in $\Gamma^{\prime}$ if for all subsets $K$ of players with $1 \leq|K| \leq k$, all strategy profiles $\vec{\sigma}$ in $\Gamma$, all strategies $\sigma_{e}$ for the environment, all type profiles $\vec{x} \in \mathcal{T}$, and all players $i \in K$, we have

$$
u_{i}\left(\Gamma^{\prime}, \vec{\sigma}^{\prime}, \sigma_{e}, \vec{x}_{K}\right)>u_{i}\left(\Gamma,\left(\vec{\sigma}_{K}, \vec{\rho}_{-K}\right), \vec{x}_{K}\right) .
$$

Thus, if $\vec{\rho}$ is a $k$-punishment strategy with respect to $\vec{\sigma}^{\prime}$ and all but $k$ players play their part of $\vec{\rho}$ in the underlying tame, then all of the remaining players will be worse off than they would be in $\Gamma^{\prime}$ if everyone had played $\vec{\sigma}^{\prime}$, no matter what they do in the underlying game.

THEOREM 4.4. If $\Gamma$ is a normal-form Bayesian game with $n$ players, $\vec{\sigma}+\sigma_{d}$ is a strategy profile in canonical form for the players and mediator in an asynchronous mediator game $\Gamma_{d}$ that extends $\Gamma$, there is a $(k+t)$-punishment strategy with respect to $\vec{\sigma}+\sigma_{d}$, and $n>3 k+4 t$, then with the AH approach, there exists a strategy profile $\vec{\sigma}_{C T}$ that implements $\vec{\sigma}+\sigma_{d}$ in the asynchronous cheap-talk game $\Gamma_{C T}$, and if $\vec{\sigma}+\sigma_{d}$ is a (strongly) $(k, t)$-robust equilibrium in $\Gamma_{d}$, then $\vec{\sigma}_{C T}$ is a (strongly) $(k, t)$-robust equilibrium in $\Gamma_{C T}$. If we require only that $\vec{\sigma}_{C T}$ is a weak implementation, then the number of messages in a history of $\vec{\sigma}_{C T}$ is polynomial in $n$ and linear in $c$.

Note that in Theorem 4.4, the running time of the algorithm is significantly affected by whether we want $\vec{\sigma}_{C T}$ to implement $\vec{\sigma}$ or whether a weak implementation suffices.

If we assume both that there is a $(2 k+2 t)$-punishment strategy and that utilities are known, we can get an analogue to R2, but with an $\epsilon$ error.

THEOREM 4.5. If $\Gamma$ is a normal-form Bayesian game with $n$ players, $\vec{\sigma}+\sigma_{d}$ is a strategy profile in canonical form for the players and mediator in an asynchronous mediator game $\Gamma_{d}$ that extends $\Gamma$, there is $a(2 k+2 t)$-punishment strategy with respect to $\vec{\sigma}+\sigma_{d}$, and $n>2 k+3 t$, then with the AH approach, for all $\epsilon>0$ there is a strategy $\vec{\sigma}_{C T}$ that $\epsilon$-implements $\vec{\sigma}$ in the asynchronous cheap-talk game $\Gamma_{C T}$ such that if $\vec{\sigma}+\sigma_{d}$ is a (strongly) $(k, t)$-robust equilibrium in $\Gamma_{d}$, then $\vec{\sigma}_{C T}$ is a (strongly) $\epsilon-(k, t)$-robust equilibrium in $\Gamma_{C T}$. If we require only that $\vec{\sigma}_{C T}$ is a weak implementation, then the number of messages in a history of $\vec{\sigma}_{C T}$ is polynomial in $n$ and linear in $c$.

We prove these results using ideas in the spirit of $\mathrm{ADGH}$, but much more care must be taken to deal with asynchrony. Among other things, we need stronger security guarantees than are traditionally provided for multiparty communication; see Section 5 for details.

\section{BISIMULATION AND COTERMINATION}

Ben-Or, Goldwasser, and Wigderson [7] (BGW from now on) and Ben-Or, Canetti, and Goldreich [6] (BCG from now on) show that if a function $f$ of $n$ inputs provided by $n$ players can be computed using a mediator, then it can be computed by the players without the mediator and without revealing any information beyond the function value, even when some of the players are malicious. BGW deal with the synchronous case and provide a protocol that tolerates up to $n / 3$ malicious players; BCG deal with the asynchronous case and provide a protocol that tolerates up to $n / 4$. The notion of not revealing any information is made precise by defining a set of ideal distributions over possible values of the function, and ensuring that the real distribution is identically distributed to one of those (see BGW and BCG for formal definitions and details).

We can view a mediator game as computing an action profile in the underlying game; the ideal distributions are the possible distributions over action profiles when the honest players play their component of the $(k, t)$-robust equilibrium strategy profile in the mediator game. BCG's protocol then essentially gives us a strategy in the cheap-talk game. However, the BCG protocol is not sufficient for our purposes for two reasons: it does not guarantee that the real protocol is an implementation of the ideal protocol in the sense of the definition in Section 2 (although it does suffice for weak implementation), nor does it guarantee that the protocol is a $(k, t)$-robust equilibrium. To prove these stronger results, we show that $\vec{\sigma}_{C T}$ can be constructed so as to satisfy some additional security properties, which we now define. For some of these definitions, it will be useful to introduce the notion of adversary. An adversary $A$ is a tuple $\left(T, \vec{\tau}_{T}, \sigma_{e}\right)$ consisting of a set $T$ of malicious players, a strategy $\vec{\tau}_{T}$ for players in $T$, and a strategy $\sigma_{e}$ for the scheduler. To keep the notation as simple as possible, we omit the set $T$ when it is clear from context, just writing $A$ as the pair $\left(\vec{\tau}_{T}, \sigma_{e}\right)$. (Note that $\vec{\tau}_{T}$ is just a tuple of $|T|$ strategies. We write this tuple as $\vec{\tau}_{T}$ only because we view it as the strategies played by the players in $T$ in the profile $\vec{\tau}$.)

Definition 5.1 ( $t$-Bisimulation). Let $\vec{\pi}(\vec{x}, A)$ be the distribution over outputs when running strategy $\vec{\pi}$ with adversary $A=\left(\tau_{T}, \sigma_{e}\right)$. Protocol $\vec{\pi}^{\prime} t$-bisimulates $\vec{\pi}+\pi_{d}$ if, for all $T$ with $|T| \leq t$ and inputs $\vec{x}$ :

- for all adversaries $A=\left(\vec{\tau}_{T}, \sigma_{e}\right)$, there exists an adversary $A^{\prime}=\left(\vec{\tau}_{T}^{\prime}, \sigma_{e}^{\prime}\right)$ such that $\vec{\pi}(\vec{x}, A)$ and $\vec{\pi}^{\prime}\left(\vec{x}, A^{\prime}\right)$ identically distributed;

- for all adversaries $A^{\prime}=\left(\vec{\tau}_{T}^{\prime}, \sigma_{e}^{\prime}\right)$, there exists an adversary $A=\left(\vec{\tau}_{T}, \sigma_{e}\right)$ such that $\vec{\pi}(\vec{x}, A)$ and $\vec{\pi}^{\prime}\left(\vec{x}, A^{\prime}\right)$ are identically distributed.

Note that 0 -bisimulation is equivalent to implementation. While implementation considers only what happens when there is no malicious behaviour, $t$-bisimulation generalizes this notion by taking malicious behavior into account. For some of our results (specifically, Theorems 4.1 and 4.2), we use this notion, and show that it is achievable under the conditions of these theorems. 
We have required schedulers to eventually deliver each message that is sent. Because we assume that protocols in the mediator game are bounded, all protocols in the mediator game must terminate. This means that we can't hope to simulate a protocol in the cheaptalk game that deadlocks. (We assume that if the protocol deadlocks, it has a special output that we denote $\perp$. Given our constraints, we can never get an output of $\perp$ in the mediator game.) To deal with this situation, we relax this requirement on schedulers somewhat, but only in the mediator game. We take a relaxed scheduler to be one that may never deliver some messages. There is no requirement on messages sent by the players. We can define relaxed $t$-bisimulation just as we defined $t$-bisimulation, except that we allow the schedulers $\sigma_{e}^{\prime}$ and $\sigma_{e}$ to be relaxed schedulers. Finally, we define $\left(t, t^{\prime}\right)$-bisimulation just as we defined relaxed $t$-bisimulation except that $\sigma_{e}^{\prime}$ and $\sigma_{e}$ must be standard if $|T| \leq t^{\prime}$ (note that $t^{\prime}$ must be smaller than $t$ ).

We need a further property to deal with protocols that involve punishment strategies. For a punishment strategy to be effective, all the honest players have to play it. In our protocols, the punishment strategy is played when there is a deadlock (so some players never terminate); that is, the punishment strategy is in the honest players' "wills". Thus, we want it to be the case that either none of the honest players terminate (in which case the punishment strategy will be effective) or all of them do; we do not want it to be the case that only some of the honest players terminate.

Definition $5.2\left(\left(t, t^{\prime}\right)\right.$-COtermination). A protocol $\vec{\pi}\left(t, t^{\prime}\right)$ coterminates if, for all schedulers $\sigma_{e}$, all subsets $T$ of at most $t$ players, and all strategy profiles $\vec{\tau}_{T}$ for the players in $T$, in all histories of the protocol $\left(\vec{\pi}_{-T}, \vec{\tau}_{T}, \sigma_{e}\right)$, either all the players not in $T$ terminate, or at most $t^{\prime}$ players not in $T$ do. We say that $\vec{\pi} t$-coterminates if it $(t, 0)$-coterminates.

For some of our results, we need "approximate" versions of $t$ bisimulation, relaxed $t$-bisimulation, $\left(t, t^{\prime}\right)$ bisimulation, $t$-cotermination and $\left(t, t^{\prime}\right)$-cotermination, that allow an $\epsilon$ probability of error. For $t$-bisimulation, relaxed $t$-bisimulation, and $\left(t, t^{\prime}\right)$ bisimulation, this means that the distance between the distribution over outputs in the cheap-talk game and the distribution over outputs in the mediator game is at most $\epsilon$ (where the notion of distance is that used in the definition of $\epsilon$-implementation in Section 2), while for $t$-cotermination and $\left(t, t^{\prime}\right)$-cotermination it means that the property holds with probability $1-\epsilon$. We call these properties $\epsilon$ - $t$-bisimulation, relaxed $\epsilon$ - $t$-bisimulation, $\epsilon-\left(t, t^{\prime}\right)$-bisimulation, $\epsilon-t$ cotermination, and $\epsilon-\left(t, t^{\prime}\right)$-cotermination respectively. In our companion paper [11], we prove the following results:

THEOREM 5.3. Given a mediator game $\Gamma_{d}$ extending $\Gamma$ and a strategy profile $\vec{\sigma}+\sigma_{d}$ in $\Gamma_{d}$ in canonical form, there exists a strategy profile $\vec{\sigma}_{C T}$ for $\Gamma_{C T}$ such that $\vec{\sigma}_{C T}(t, 2 t+1)$-coterminates and $t$ bisimulates (resp., $\left(t, t^{\prime}\right)$-bisimulates) $\vec{\sigma}+\sigma_{d}$ if $t<n / 3$ and $t<n / 4$ (resp., $3 t+t^{\prime}<n$ ) respectively. If $\sigma_{d}$ is responsive, the number of messages sent in a history of $\vec{\sigma}_{C T}$ is polynomial in $n$ and $N$, linear in $c$, and independent of $\vec{u}^{\prime}$.

TheOREM 5.4. Given a mediator game $\Gamma_{d}$ extending $\Gamma$, a strategy profile $\vec{\sigma}+\sigma_{d}$ in $\Gamma_{d}$ in canonical form, and a real number $\epsilon \in(0,1]$, there exists a strategy profile $\vec{\sigma}_{C T}$ in $\Gamma_{C T}$ such that $\vec{\sigma}_{C T} \epsilon-(t, t+1)$ coterminates and $\epsilon$ - $t$-bisimulates (resp., $\epsilon-\left(t, t^{\prime}\right)$-bisimulates) $\vec{\sigma}+\sigma_{d}$ if $t<n / 2$ and $t<n / 3$ (resp., $2 t+t^{\prime}<n$ ) respectively. If $\sigma_{d}$ is responsive, the number of messages sent in a history of $\vec{\sigma}_{C T}$ is polynomial in $n$ and $N$, linear in $c$, and independent of $\vec{u}^{\prime}$.

We use the constructions provided by Theorems 5.3 and 5.4 to prove Theorems 4.1, 4.2, 4.4, and 4.5. For Theorems 4.1 and 4.2, we show that these constructions already satisfy all the desired properties. For Theorems 4.4 and $4.5,(t+k, t)$-bisimulation and $\epsilon-(t+k, t)$-bisimulation guarantee that that the only way in which rational and malicious players can make the outcome of the cheaptalk game different from that of the mediator game is by preventing the honest players from terminating. However, it is not in the interest of rational players that too many honest players end in deadlock, since if enough honest players do not terminate, they play the punishment strategy according to their wills.

We then show that the construction for Theorems 5.3 and 5.4 can be easily modified so that $\vec{\sigma}_{C T}$ terminate or none does. It would then seem that to always be in the interest of rational players that all honest players terminate. Unfortunately, a punishment strategy payoff is worse for rational players than the equilibrium payoff only in expectation, and thus, depending on the information rational players receive throughout the game, there might be some situations in which they might actually want to be punished (we provide a concrete example in the full paper). We deal with this issue by showing that all mediator games can be reduced to a mediator game where the mediator uses a minimally-informative strategy, so that players receive no information about the outcome until the very end. This makes the punishment strategy a persistent threat for rational players. The proof of Theorem 4.1 is given in Section 6.3, while the proofs of Theorems 4.2, 4.4 and 4.5 can be found in the full paper.

\section{PROOFS OF THE MAIN THEOREMS}

In this section, we sketch the proof of the main theorems. The details can be found in the full paper [1].

\subsection{Coordination between the environment and malicious players}

Before proving the main results, it is useful to understand some of the implication of $(k, t)$-robustness, particularly when it comes to the interactions between the environment and the malicious and rational players. The definition of $(k, t)$-robustness requires that rational players have no profitable deviation, no matter what the malicious players and the environment do. It may seem $a$ priori that the malicious players, the rational players, and the environment all act independently, but in fact, we can assume without loss of generality that they are all under the control of a single adversary. Clearly rational players can coordinate by sending messages to each other. The malicious and rational players can also coordinate with the environment so that, for example, the malicious and rational players can act knowing who will be scheduled when and the environment can schedule rational and malicious players based on their inputs. This follows from the fact that $(k, t)$-robustness must hold for all schedulers.

To see that a player $i$ can communicate with the environment, recall that we have assumed that the message space is finite, say 
$\left\{m_{0}, \ldots, m_{M}\right\}$. Immediately after sending $m_{j}, i$ sends $j$ empty messages to itself. So, even though the environment cannot read the messages, it will know that $i$ sent message $m_{j}{ }^{2}$ (Clearly the environment will also know who sent the message, since the environment delivered the message.) Thus, we can assume without loss of generality that the rational and malicious players know the environment's protocol (and thus know when a message that is sent will be delivered), hence it suffices for the environment to tell the non-honest players when the $k$ th message is sent, who sent it, and who the intended recipient is. All the non-honest players $i$ initially send themselves $(n+1)^{2}$ empty messages. If the first message was sent by player $j_{1}$ to player $j_{2}$ (treating the mediator as player 0 in the mediator game), then the environment delivers $(n+1) j_{1}+j_{2}$ of these empty messages. Then player $i$ sends another $(n+1)^{2}$ empty messages, allowing the environment to encode the sender and receiver of the next message, if there is one.

The fact that the environment and the malicious players can communicate allows us to prove a tighter correspondence between deviations in the cheap-talk game and deviations in the mediator game than the one given by $t$-bisimulation.

Proposition 6.1. Given two protocols $\vec{\pi}$ and $\vec{\pi}^{\prime}$ and a scheduler $\sigma_{e}$, if $\vec{\pi}^{\prime} t$-bisimulates $\vec{\pi}$, there exists a function $H_{\sigma_{e}}$ from strategies to strategies such that $H_{\sigma_{e}}\left(\vec{\pi}_{i}\right)=\vec{\pi}_{i}^{\prime}$ for all $i$, and for all adversaries $A=\left(\vec{\tau}_{T}, \sigma_{e}\right)$ with $|T| \leq t$, there exists a scheduler $\sigma_{e}^{\prime}$ such that for all inputs $\vec{x}, \vec{\pi}(\vec{x}, A)$ and $\vec{\pi}^{\prime}\left(\vec{x},\left(H_{\sigma_{e}}\left(\vec{\tau}_{T}\right), \sigma_{e}^{\prime}\right)\right)$ are identically distributed (where we extend $H_{\sigma_{e}}$ to strategy profiles by taking $\left.H_{\sigma_{e}}\left(\tau_{1}, \ldots, \tau_{m}\right)=\left(H_{\sigma_{e}}\left(\tau_{1}\right), \ldots, H_{\sigma_{e}}\left(\tau_{m}\right)\right)\right)$.

Proof. Since $\vec{\pi}^{\prime} t$-bisimulates $\vec{\pi}+\pi_{d}$, for each adversary $A=$ $\left(\vec{\tau}_{T}, \sigma_{e}\right)$, there exists an adversary $A^{\prime}=\left(\vec{\tau}_{T}^{\prime}, \sigma_{e}^{\prime}\right)$ such that $\vec{\pi}(\vec{x}, A)$ and $\vec{\pi}^{\prime}\left(\vec{x}, A^{\prime}\right)$ are identically distributed for all inputs $\vec{x}$. This means that, fixing $\sigma_{e}$, there exists a well-defined function $H_{\sigma_{e}}^{a d v}$ from strategy profiles to adversaries such that for all subsets $T$ with $|T| \leq$ $t$ and all strategies $\vec{\tau}_{T}$ for players in $T$, there exists a scheduler $\sigma_{e}^{\prime}$ such that $\left(\vec{\pi}+\pi_{d}\right)\left(\vec{x},\left(\vec{\tau}_{T}, \sigma_{e}\right)\right)$ and $\vec{\pi}^{\prime}\left(\vec{x}, H_{\sigma_{e}}^{a d v}\left(\vec{\tau}_{T}\right)\right)$ for all inputs $\vec{x}$ Given an adversary $A=\left(\vec{\tau}_{T}, \sigma_{e}\right)$, consider the following adversary $A^{\prime}=\left(\vec{\tau}_{T}^{\prime}, \sigma_{e}^{\prime}\right)$ :

(1) The scheduler begins the game by scheduling all players in $T$ once.

(2) Each player $i \in T$ sends the description of the strategy $\vec{\tau}_{i}$ to the scheduler $\sigma_{e}^{\prime}$ the first time it is scheduled.

(3) After receiving the strategies of the players in $T$, the scheduler computes $H_{\sigma_{e}}^{a d v}\left(\vec{\tau}_{T}\right)$ and sends each player $i \in T$ the (description of) strategy $H_{\sigma_{e}}^{a d v}\left(\vec{\tau}_{T}\right)_{i}$, and then switches to using $H_{\sigma_{e}}^{a d v}\left(\vec{\tau}_{T}\right)_{e}$.

(4) Each player $i \in T$ switches to the strategy sent by the scheduler after receiving it.

Consider the function $H_{\sigma_{e}}$ that maps $\tau_{i}$ to $\tau_{i}^{\prime}$ if $\tau_{i} \neq \pi_{i}$ and maps $\pi_{i}$ to $\pi_{i}^{\prime}$ (note that $H_{\sigma_{e}}$ is well defined, since $\tau_{i}^{\prime}$ does not depend on the strategy of other players in $T$ ) and a scheduler $\sigma_{e}^{\prime \prime}$ that acts like $\sigma_{e}^{\prime}$ except that at step 1 , it schedules only the players $i$ in $T$ such that $\tau_{i} \neq \pi_{i}$. We have by construction that $\vec{\pi}(\vec{x}, A)=\vec{\pi}^{\prime}\left(\vec{x}, H_{\sigma_{e}}^{a d v}\left(\vec{\tau}_{T}\right)\right)$ and therefore that $\vec{\pi}(\vec{x}, A)=\vec{\pi}^{\prime}\left(\vec{x},\left(H_{\sigma_{e}}\left(\vec{\tau}_{T}\right), \sigma_{e}^{\prime \prime}\right)\right)$, as desired. $\square$

\footnotetext{
${ }^{2}$ We can encode $m_{j}$ using using fewer messages by having $i$ send message to other agents as well as itself. Our goal here is not to minimize the number of messages, but to show that communication with the environment is possible.
}

Proposition 6.1 implies that we can assume without loss of generality that individual deviations in the mediator game correspond to individual deviations in the cheap-talk game and vice-versa. An analogous result holds for $\epsilon$ - $t$-bisimulation:

Proposition 6.2. Given two protocols $\vec{\pi}$ and $\vec{\pi}^{\prime}$ and a scheduler $\sigma_{e}$, if $\vec{\pi}^{\prime} \epsilon$-t-bisimulates $\vec{\pi}$, there exists a function $H_{\sigma_{e}}$ from strategies to strategies such that $H_{\sigma_{e}}\left(\vec{\pi}_{i}\right)=\vec{\pi}_{i}^{\prime}$ for all $i$, and for all adversaries $A=\left(\vec{\tau}_{T}, \sigma_{e}\right)$ with $|T| \leq t$, there exists a scheduler $\sigma_{e}^{\prime}$ such that for all inputs $\vec{x}$, the distance between the distributions $\vec{\pi}(\vec{x}, A)$ and $\vec{\pi}^{\prime}\left(\vec{x},\left(H_{\sigma_{e}}\left(\vec{\tau}_{T}\right), \sigma_{e}^{\prime}\right)\right)$ is at most $\epsilon$ (where the notion of distance is that used in the definition of $\epsilon$-implementation in Section 2).

As we show next, since the scheduler can collude with malicious players, $t$-immune strategy profiles satisfy an even stronger condition: deviations by players in a set $T$ with $|T| \leq t$ do not make things worse for the non-deviating players even if the environment colludes with the players in $T$.

Proposition 6.3. If $\vec{\sigma}$ is $t$-immune, then for all sets $T$ of players with $|T| \leq t$, strategies $\sigma_{e}$ and $\sigma_{e}^{\prime}$ for the environment, strategy profiles $\vec{\tau}_{T}$ for the players in $T$, input profiles $\vec{x}$ and $\vec{x}^{\prime}$, and players $i \notin T$, we have

$$
u_{i}\left(\Gamma_{d},\left(\vec{\sigma}_{-T}, \vec{\tau}_{T}\right), \sigma_{e}^{\prime}, \vec{x}_{T}^{\prime}\right) \geq u_{i}\left(\Gamma_{d}, \vec{\sigma}, \sigma_{e}, \vec{x}_{T}\right) .
$$

Proof. Clearly, if (1) holds for all $\sigma_{e}, \sigma_{e}^{\prime}, \vec{x}$, and $\vec{x}^{\prime}$, then $\vec{\sigma}$ is $t$-immune. For the converse, suppose by way of contradiction that $\vec{\sigma}$ is $t$-immunte but for some $T, \vec{\tau}, \sigma_{e}, \sigma_{e}^{\prime}$, and $i \notin T$, (1) does not hold. Consider a scheduler $\sigma_{e}^{\prime \prime}$ that acts just like $\sigma_{e}$, except that if some player $i$ sends a message to itself it acts like $\sigma_{e}^{\prime}$. Then players in $T$ can effectively decrease $i$ 's payoff with scheduler $\sigma_{e}^{\prime \prime}$ by sending a message to themselves and playing as if they had input $\vec{x}_{T}^{\prime}$; that is, there is a strategy $\vec{\tau}_{T}^{\prime}$ such that

$$
\begin{aligned}
& u_{i}\left(\Gamma_{d},\left(\vec{\sigma}_{-T}, \vec{\tau}_{T}^{\prime}\right), \sigma_{e}^{\prime \prime}, \vec{x}_{T}\right) \\
= & u_{i}\left(\Gamma_{d},\left(\vec{\sigma}_{-T}, \vec{\tau}_{T}\right), \sigma_{e}^{\prime}, \vec{x}_{T}^{\prime}\right) \\
<\quad & u_{i}\left(\Gamma_{d}, \vec{\sigma}, \sigma_{e}, \vec{x}_{T}\right) \\
= & u_{i}\left(\Gamma_{d}, \vec{\sigma}, \sigma_{e}^{\prime \prime}, \vec{x}_{T}\right),
\end{aligned}
$$

contradicting the assumption that $\vec{\sigma}$ is $t$-immune.

A similar argument shows that $(k, t)$-robust strategy profiles satisfy a correspondingly stronger condition, made precise in the following proposition:

Proposition 6.4. A strategy profile $\vec{\sigma}$ is $(k, t)$-robust (resp., strongly $(k, t)$-robust) if and only if it is $t$-immune and for all disjoint sets $K$ and $T$ with $1 \leq|K| \leq k$ and $|T| \leq t$, all strategy profiles $\vec{\tau}_{K}, \vec{\tau}_{T}$, and $\vec{\tau}_{T}^{\prime}$ for the players in $K$ and $T$, respectively, all environment strategies $\sigma_{e}$ and $\sigma_{e}^{\prime}$, and all input profiles $\vec{x}$ and $\vec{x}^{\prime}$, we have that

$$
\begin{aligned}
& u_{i}\left(\Gamma_{d},\left(\vec{\sigma}_{-(K \cup T)}, \vec{\tau}_{K}, \vec{\tau}_{T}^{\prime}\right), \sigma_{e}^{\prime}, \vec{x}_{(K \cup T)}^{\prime}\right) \\
\leq \quad & u_{i}\left(\Gamma_{d},\left(\vec{\sigma}_{-(K \cup T)}, \vec{\tau}_{K}, \vec{\tau}_{T}^{\prime}\right), \sigma_{e}^{\prime}, \vec{x}_{(K \cup T)}^{\prime}\right)
\end{aligned}
$$

for some $i \in K$ (resp., for all $i \in K$ ).

Proof. Again, it is clear that if (2) holds for all $K$ and $T$ with $1 \leq|K| \leq k$ and $|T| \leq t$, all $\vec{\tau}_{K}, \vec{\tau}_{T}, \vec{\tau}_{T}^{\prime}, \vec{x}$, and $\vec{x}^{\prime}$, and some (resp., all) $i \in K$, then $\vec{\sigma}$ is $(k, t)$-robust (resp., strongly ( $k, t)$-robust).

For the converse, assume by way of contradiction that $\vec{\sigma}$ is $(k, t)$ robust, but for some disjoint sets $K$ and $T$ with $1 \leq|K| \leq k$ and $|T| \leq t, \vec{\tau}_{K}, \vec{\tau}_{T}, \vec{\tau}_{T}^{\prime}, \vec{x}$, and $\vec{x}^{\prime}$, and all $i \in K$, (2) does not hold. Again, 
we use the fact that the rational players can effectively communicate with malicious players and with the scheduler. Consider a scheduler $\sigma_{e}^{\prime \prime}$ that acts like $\sigma_{e}$ unless some player sends a message to itself, in which case it acts like $\sigma_{e}^{\prime}$, and a strategy profile $\vec{\tau}_{T}^{\prime \prime}$ in which each player $i \in T$ acts as if it was using strategy $\left(\tau_{T}\right)_{i}$, except that it switches to $\left(\tau_{T}^{\prime}\right)_{i}$ and acts as if it has input $x_{i}^{\prime}$ if it receives a message from a rational player (i.e., a player in $K$ ) asking it to do so. Then, given input profile $\vec{x}$, strategy profile $\vec{\tau}_{T}^{\prime \prime}$ for $T$, and scheduler $\sigma_{e}^{\prime \prime}$, player $i$ can gain by sending a message to itself and sending a message to players in $T$ asking them to follow $\vec{\tau}_{T}^{\prime}$ and to act is if they have input $\vec{x}_{T}^{\prime}$, and by having players in $K$ play $\vec{\tau}_{K}$ as if they had input $\vec{x}_{K}^{\prime}$, rather than playing $\vec{\sigma}$. This contradicts the assumption that $\vec{\sigma}$ is $(k, t)$-robust. The argument for strong $(k, t)$-robustness is analogous.

Another property interesting in its own right that follows from this argument is that $(k, t)$-robust strategies must be schedulerproof: the expected payoff for all players is the same regardless of the scheduler:

COROLLARY 6.5. If $\vec{\sigma}$ is $(k, t)$-robust for some $k \geq 1$, then for all sets $T$ with $|T| \leq t$, strategy profiles $\vec{\tau}_{T}$ for the players in $T$, environment strategies $\sigma_{e}$ and $\sigma_{e}^{\prime}$, input profiles $\vec{x}$, and players $i \notin T$, we have $u_{i}\left(\Gamma,\left(\vec{\sigma}_{-T}, \vec{\tau}_{T}\right), \sigma_{e}, \vec{x}_{T}\right)=u_{i}\left(\Gamma,\left(\vec{\sigma}_{-T}, \vec{\tau}_{T}\right), \sigma_{e}^{\prime}, \vec{x}_{T}\right)$.

We have analogous strengthenings of $\epsilon$ - $t$-immunity and $\epsilon-(k, t)$ robustness, which are stated next. The proofs are essentially identical to that of Proposition 6.3 and 6.4 respectively, so we omit them here.

Proposition 6.6. If $\epsilon>0$ and $\vec{\sigma}$ is $\epsilon$-t-immune in game $\Gamma$, then for all sets $T$ of players with $|T| \leq t$, strategy profiles $\tau_{T}$ for the players in T, environment strategies $\sigma_{e}$ and $\sigma_{e}^{\prime}$, input profiles $\vec{x}$ and $\vec{x}^{\prime}$, and players $i \notin T$, we have that

$$
u_{i}\left(\Gamma,\left(\vec{\sigma}_{-T}, \vec{\tau}_{T}\right), \sigma_{e}^{\prime}, \vec{x}_{T}^{\prime}\right)>u_{i}\left(\Gamma, \vec{\sigma}, \sigma_{e}, \vec{x}_{T}\right)-\epsilon .
$$

Proposition 6.7. A strategy profile $\vec{\sigma}$ is $\epsilon-(k, t)$-robust (resp., strongly $\epsilon-(k, t)$-robust) in game $\Gamma$ if and only if it is $\epsilon$-t-immune and, for all disjoint sets $K$ and $T$ of players with $1 \leq|K| \leq k$ and $|T| \leq t$, all strategy profiles $\vec{\tau}_{K}, \vec{\tau}_{T}^{\prime}$, and $\vec{\tau}_{T}$ for players in $K$ and $T$, respectively, all environment strategies $\sigma_{e}$ and $\sigma_{e}^{\prime}$, and all input profiles $\vec{x}$ and $\vec{x}^{\prime}$, we have that

$u_{i}\left(\Gamma,\left(\vec{\sigma}_{-(K \cup T)}, \vec{\tau}_{K}, \vec{\tau}_{T}^{\prime}\right), \sigma_{e}^{\prime}, \vec{x}_{(K \cup T)}^{\prime}\right)<u_{i}\left(\Gamma,\left(\vec{\sigma}_{-T}, \vec{\tau}_{T}\right), \sigma_{e}, \vec{x}_{T}\right)+\epsilon$ for some $i \in K$ (resp., for all $i \in K$ ).

It will be useful for our later results that we can actually improve on the bound of $\epsilon$ in Propositions 6.6 and 6.7.

Proposition 6.8. If $\vec{\sigma}$ is an $\epsilon$-t-immune strategy in a finite game $\Gamma$, then there exists $\epsilon_{0}$ with $0<\epsilon_{0}<\epsilon$ such that for all sets of players $T$ with $|T| \leq t$, strategy profiles $\vec{\tau}_{T}$ for the players in $T$, environment strategies $\sigma_{e}$ and $\sigma_{e}^{\prime}$, input profiles $\vec{x}$ and $\vec{x}^{\prime}$, and players $i \notin T$, we have that

$$
u_{i}\left(\Gamma,\left(\vec{\sigma}_{-T}, \vec{\tau}_{T}\right), \sigma_{e}^{\prime}, \vec{x}_{T}^{\prime}\right)>u_{i}\left(\Gamma, \vec{\sigma}, \sigma_{e}, \vec{x}_{T}\right)-\epsilon_{0} .
$$

Proof. Since, by Proposition 6.6, for each choice of $\vec{\tau}_{T}, \sigma_{e}$, and $\sigma_{e}^{\prime}$, we have

$$
u_{i}\left(\Gamma, \vec{\sigma}, \sigma_{e}, \vec{x}_{T}\right)-u_{i}\left(\Gamma,\left(\vec{\sigma}_{-T}, \vec{\tau}_{T}\right), \sigma_{e}^{\prime}, \vec{x}_{T}^{\prime}\right)<\epsilon,
$$

and the space of player strategy profiles, environment strategies, and input value profiles is compact, if we take the sup of the lefthand side over all choices of strategy profiles $\vec{\tau}_{T}$, environment strategies $\sigma_{e}$ and $\sigma_{e}^{\prime}$, and input profiles $\vec{x}$ and $\vec{x}^{\prime}$, it takes on some maximum value $\epsilon_{1}<\epsilon_{0}$. We can then take $\epsilon_{0}=\left(\epsilon+\epsilon_{1}\right) / 2$.

Using Proposition 6.7, we get a similar result for $\epsilon$ - $(k, t)$-robustness. The proof is analogous to that of Proposition 6.8.

Proposition 6.9. If $\Gamma$ is a finite game and $\vec{\sigma}$ is a $\epsilon-(k, t)$-robust strategy (resp., strongly $\epsilon-(k, t)$-robust strategy) in $\Gamma_{d}$, then there exists $\epsilon_{0}$ with $0<\epsilon_{0}<\epsilon$ such that for all disjoint sets $K$ and $T$ of players with $1 \leq|K| \leq k$ and $|T| \leq t$, all strategy profiles $\vec{\tau}_{K}, \vec{\tau}_{T}$ and $\vec{\tau}_{T}^{\prime}$ for players in $K$ and $T$, respectively, all environment strategies $\sigma_{e}$ and $\sigma_{e}^{\prime}$, and all input profiles $\vec{x}$ and $\vec{x}^{\prime}$, we have that

$u_{i}\left(\Gamma,\left(\vec{\sigma}_{-(K \cup T)}, \vec{\tau}_{K}, \vec{\tau}_{T}\right), \sigma_{e}, \vec{x}_{(K \cup T)}^{\prime}\right)<u_{i}\left(\Gamma,\left(\vec{\sigma}_{-T}, \vec{\tau}_{T}^{\prime}\right), \sigma_{e}^{\prime}, \vec{x}_{T}\right)+\epsilon_{0}$

for some $i \in K$ (resp., all $i \in K)$.

\subsection{Constructing a protocol that $t$-coterminates}

If $t<n / 3$ we can get an an analogue of Theorems 5.3 and 5.4 by replacing $(t, 2 t+1)$-cotermination by $t$-cotermination and $\epsilon-(t, t+$ 1)-cotermination by $\epsilon$ - $t$-cotermination respectively. We sketch the construction for $t$-cotermination; an analogous construction achieves $\epsilon$ - $t$-cotermination.

Consider a protocol $\vec{\sigma}_{C T}^{\prime}$ in which players play just as in $\vec{\sigma}_{C T}$ except that, whenever an honest player $i$ terminates in $\vec{\sigma}_{C T}$, it instead broadcasts an 'OK' message to all players and waits until it receives $3 t+1$ 'OK' messages before it terminates in $\vec{\sigma}_{C T}^{\prime}$. Note that if an honest player terminates in $\vec{\sigma}_{C T}^{\prime}$, then at least $3 t+1$ players must have broadcast an 'OK' message in this history of $\vec{\sigma}_{C T}^{\prime}$, of which at least $2 t+1$ are honest. Thus, at least $2 t+1$ players terminate in the corresponding history of $\vec{\sigma}_{C T}$. Since $\vec{\sigma}_{C T}(t, 2 t+1)$-coterminates, it follows that all players not in $T$ must terminate with $\vec{\sigma}_{C T}$, and hence all players not in $T$ send an 'OK' message (and terminate) with $\vec{\sigma}_{C T}^{\prime}$.

It remains to show that $\vec{\sigma}_{C T}^{\prime}$ still $\left(t, t^{\prime}\right)$-bisimulates $\vec{\sigma}+\sigma_{d}$ if $3 t+t^{\prime}<n$. Clearly, it still relaxed $t$-bisimulates $\vec{\sigma}+\sigma_{d}$, so we just have to show that all players are guaranteed to terminate in the presence of at most $t^{\prime}$ malicious players. However, in this case, by assumption, all honest players are guaranteed to terminate in $\vec{\sigma}_{C T}$, and thus all honest players are guaranteed to eventually send an 'OK' broadcast in $\vec{\sigma}_{C T}^{\prime}$. Since $n-t^{\prime}>3 t+1$, this guarantees that there will be at least $3 t+1$ 'OK' broadcasts and all honest players will eventually terminate, as desired.

Note that this construction requires players a reliable broadcast protocol, and thus can be done only if $n>3(t+k)$. To prove Theorem 4.5 we require different techniques.

\subsection{Proof of Theorem 4.1}

By Theorem 5.3, if $n>4 k+4 t$, there exists a strategy profile $\vec{\sigma}_{C T}$ that $(k+t)$-bisimulates $\vec{\sigma}+\sigma_{d}$. It is immediate from the definition of $(k+t)$-bisimulation that $\vec{\sigma}_{C T}$ implements $\vec{\sigma}+\sigma_{d}$. Since the probability of deadlock is 0 , what the players do in case of deadlock is irrelevant, so this approach works both in the case of the $\mathrm{AH}$ approach and the default-move approach. It remains to show that, 
for each utility variant $\Gamma_{d}\left(\vec{u}^{\prime}\right)$ of $\Gamma_{d}$, if $\vec{\sigma}+\sigma_{d}$ is a (strongly) $(k, t)$ robust equilibrium in $\Gamma_{d}\left(\vec{u}^{\prime}\right)$, then $\vec{\sigma}_{C T}$ is a (strongly) $(k, t)$-robust equilibrium in $\Gamma_{C T}\left(\vec{u}^{\prime}\right)$. We start by showing that $\vec{\sigma}_{C T}$ is $t$-resilient in $\Gamma_{C T}\left(\vec{u}^{\prime}\right)$.

Given $T$ with $|T| \leq t, \vec{\tau}_{T}$, and $\sigma_{e}$, by Theorem 5.3 and Proposition 6.1, there exists a function $H_{\sigma_{e}}$ from strategies to strategies and a scheduler $\sigma_{e}^{\prime}$ such that for all input profiles $\vec{x}$,

$$
\begin{aligned}
& u_{i}^{\prime}\left(\Gamma_{C T}\left(\vec{u}^{\prime}\right),\left(\left(\vec{\sigma}_{C T}\right)_{-T}, \tau_{T}\right), \sigma_{e}, \vec{x}\right) \\
= & u_{i}^{\prime}\left(\Gamma_{d}\left(\vec{u}^{\prime}\right),\left(\vec{\sigma}_{-T}, H_{\sigma_{e}}\left(\vec{\tau}_{T}\right)\right), \sigma_{e}^{\prime}, \vec{x}\right)
\end{aligned}
$$

for all players $i$. There also exists a scheduler $\sigma_{e}^{\prime \prime}$ such that

$$
u_{i}^{\prime}\left(\Gamma_{C T}\left(\vec{u}^{\prime}\right), \vec{\sigma}_{C T}, \sigma_{e}^{\prime}, \vec{x}\right)=u_{i}^{\prime}\left(\Gamma_{d}\left(\vec{u}^{\prime}\right), \vec{\sigma}, \sigma_{e}^{\prime \prime}, \vec{x}\right) .
$$

Since $\vec{\sigma}$ is $t$-immune, for all $i \notin T$ we have that

$$
\begin{array}{rlr} 
& u_{i}^{\prime}\left(\Gamma_{C T}\left(\vec{u}^{\prime}\right),\left(\left(\vec{\sigma}_{C T}\right)_{-T}, \vec{\tau}_{T}\right), \sigma_{e}, \vec{x}_{T}\right) & \\
= & u_{i}^{\prime}\left(\Gamma_{d}\left(\vec{u}^{\prime}\right),\left(\vec{\sigma}_{-T}, H_{\sigma_{e}}\left(\vec{\tau}_{T}\right)\right), \sigma_{e}^{\prime}, \vec{x}_{T}\right) & \\
\geq & u_{i}^{\prime}\left(\Gamma_{d}\left(\vec{u}^{\prime}\right), \vec{\sigma}, \sigma_{e}^{\prime \prime}, \vec{x}_{T}\right) & \text { [by Lemma 6.3] } \\
= & u_{i}^{\prime}\left(\Gamma_{C T}\left(\vec{u}^{\prime}\right), \vec{\sigma}_{C T}, \sigma_{e}^{\prime}, \vec{x}_{T}\right) . &
\end{array}
$$

Therefore, $\vec{\sigma}_{C T}$ is $t$-immune.

To show (strong) $(k, t)$-robustness, taking $\vec{\tau}_{T}, \sigma_{e}$, and $\sigma_{e}^{\prime}$ as above, suppose that $K$ is a set of players disjoint from $T$ such that $|K| \leq k$, and the players in $K$ play $\vec{\tau}_{K}$. By Theorem 5.3 and Proposition 6.1, there exists $\sigma_{e}^{*}$ and $H_{\sigma_{e}}$ such that

$$
\begin{aligned}
& u_{i}^{\prime}\left(\Gamma_{C T}\left(\vec{u}^{\prime}\right),\left(\left(\vec{\sigma}_{C T}\right)_{-(K \cup T)}, \vec{\tau}_{K}, \vec{\tau}_{T}\right), \sigma_{e}, \vec{x}\right) \\
= & u_{i}^{\prime}\left(\Gamma_{d}\left(\vec{u}^{\prime}\right),\left(\vec{\sigma}_{-(K \cup T)}, H_{\sigma_{e}}\left(\vec{\tau}_{K}\right), H_{\sigma_{e}}\left(\vec{\tau}_{T}\right)\right), \sigma_{e}^{*}, \vec{x}_{(K \cup T)}\right)
\end{aligned}
$$

for all players $i$. By Corollary 6.4, if $\vec{\sigma}+\sigma_{d}$ is $(k, t)$-robust (resp., strongly $(k, t)$-robust) in $\Gamma_{d}\left(\vec{u}^{\prime}\right)$, then

$$
\begin{aligned}
& u_{i}^{\prime}\left(\Gamma_{d}\left(\vec{u}^{\prime}\right),\left(\vec{\sigma}_{-(K \cup T)}, H_{\sigma_{e}}\left(\vec{\tau}_{K}\right), H_{\sigma_{e}}\left(\vec{\tau}_{T}\right)\right), \sigma_{e}^{*}, \vec{x}_{(K \cup T)}\right) \\
\leq \quad & u_{i}^{\prime}\left(\Gamma_{d}\left(\vec{u}^{\prime}\right),\left(\vec{\sigma}_{-T}, H_{\sigma_{e}}\left(\vec{\tau}_{T}\right)\right), \sigma_{e}^{\prime}, \vec{x}_{T}\right)
\end{aligned}
$$

for some (resp., all) $i \in K$. For those $i \in K$ for which this inequality holds, we have

$$
\begin{aligned}
& u_{i}^{\prime}\left(\Gamma_{C T}\left(\vec{u}^{\prime}\right),\left(\left(\vec{\sigma}_{C T}\right)_{-(K \cup T)}, \tau_{K}, \tau_{T}\right), \sigma_{e}, \vec{x}_{(K \cup T)}\right) \\
= & u_{i}^{\prime}\left(\Gamma_{d}\left(\vec{u}^{\prime}\right),\left(\vec{\sigma}_{-(K \cup T)}, H_{\sigma_{e}}\left(\vec{\tau}_{K}\right), H_{\sigma_{e}}\left(\vec{\tau}_{T}\right)\right), \sigma_{e}^{*}, \vec{x}_{(K \cup T)}\right) \\
\leq & u_{i}^{\prime}\left(\Gamma_{d}\left(\vec{u}^{\prime}\right),\left(\vec{\sigma}_{-T}, H_{\sigma_{e}}\left(\vec{\tau}_{T}\right)\right), \sigma_{e}^{\prime}, \vec{x}_{T}\right) \\
= & u_{i}^{\prime}\left(\Gamma_{C T}\left(\vec{u}^{\prime}\right),\left(\left(\vec{\sigma}_{C T}\right)_{-T}, \sigma_{e}, \vec{x}_{T}\right) .\right.
\end{aligned}
$$

It follows that $\vec{\sigma}_{C T}$ is (strongly) $(k, t)$-robust in $\Gamma_{C T}\left(\vec{u}^{\prime}\right)$.

\section{CONCLUSION}

We have extended the results of ADGH on implementing mediators to the asynchronous setting. This setting raises a number of new subtleties, particularly regarding how to define utilities in a game where players do not terminate. Since many real-world applications are asynchronous, and thinking in terms of mediators in this setting provides much simpler approach to designing efficient mechanisms, having a "compiler" that can translate solutions with a mediator to one without a mediator can be quite useful in principle.

There are still a number of questions that remain open. The most obvious ones involve lower bounds. Lower bounds that match the upper bounds of ADGH in the synchronous setting were proved by Abraham, Dolev, and Halpern [3]. Can we provide analogous lower bounds here? In addition, we have considered only noncryptographic setting; ADGH also provided bounds for the setting where players were polynomially-bounded and could use cyrptographic tools. To what extent do things change in this setting in the asynchronous case?

\section{REFERENCES}

[1] I. Abraham, D. Dolev, I. Geffner, and J. Y. Halpern. 2019. Implementing mediators with asynchronous cheap talk. (2019). Available at http://arxiv.org/abs/1806.01214.

[2] I. Abraham, D. Dolev, R. Gonen, and J. Y. Halpern. 2006. Distributed computing meets game theory: robust mechanisms for rational secret sharing and multiparty computation. In Proc. 25th ACM Symposium on Principles of Distributed Computing. 53-62.

[3] I. Abraham, D. Dolev, and J. Y. Halpern. 2008. Lower bounds on implementing robust and resilient mediators. In Fifth Theory of Cryptography Conference. 302319 .

[4] E. Adar and B. Huberman. 2000. Free riding on Gnutella. First Monday 5, 10 (2000).

[5] R. J. Aumann and S. Hart. 2003. Long cheap talk. Econometrica 71, 6 (2003), $1619-1660$.

[6] M. Ben-Or, R. Canetti, and O. Goldreich. 1993. Asynchronous secure computation. In STOC '93: Proceedings of the 25 Annual ACM Symposium on Theory of Computing. $52-61$.

[7] M. Ben-Or, S. Goldwasser, and A. Wigderson. 1988. Completeness theorems for non-cryptographic fault-tolerant distributed computation. In Proc. 20th ACM Symp. Theory of Computing. 1-10.

[8] M. Ben-Or, B. Kelmer, and T. Rabin. 1994. Asynchronous secure computations with optimal resilience (extended abstract). In Proc. 13th ACM Symp. Principles of Distributed Computing. 183-192.

[9] E. Ben-Porath. 2003. Cheap talk in games with incomplete information. Fournal of Economic Theory 108, 1 (2003), 45-71.

[10] S. Even, O. Goldreich, and A. Lempel. 1985. A randomized protocol for signing contracts. Commun. ACM 28, 6 (1985), 637-647. https://doi.org/10.1145/3812.3818

[11] I. Geffner and J. Y. Halpern. 2018. Stronger security guarantees for multiparty computation. (2018). Available at http://arxiv.org.

[12] O. Goldreich, S. Micali, and A. Wigderson. 1987. How to play any mental game. In Proc. 19th ACM Symp. Theory of Computing. 218-229.

[13] J. Y. Halpern and M. R. Tuttle. 1993. Knowledge, probability, and adversaries. 40, 4 (1993), 917-962.

[14] A. Shamir, R. L. Rivest, and L. Adelman. 1981. Mental poker. In The Mathematical Gardner, D. A. Klarner (Ed.). Prindle, Weber, and Schmidt, Boston, MA, 37-43.

[15] A. Yao. 1982. Protocols for secure computation (extended abstract). In Proc. 23rd IEEE Symp. Foundations of Computer Science. 160-164. 